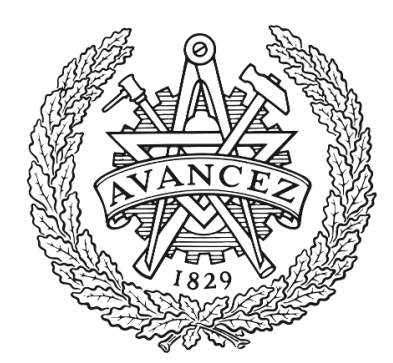

CHALMERS

\title{
Millimeter Wave E-Plane Transition From Waveguide to Microstrip Line With Large Substrate Size Related to MMIC Integration
}

Downloaded from: https://research.chalmers.se, 2023-04-26 11:23 UTC

Citation for the original published paper (version of record):

Uz Zaman, A., Vassilev, V., Kildal, P. et al (2016). Millimeter Wave E-Plane Transition From Waveguide to Microstrip Line With Large Substrate Size

Related to MMIC Integration. IEEE Microwave and Wireless Components Letters, 26(7): 481-483.

http://dx.doi.org/10.1109/lmwc.2016.2574995

N.B. When citing this work, cite the original published paper.

(O2016 IEEE. Personal use of this material is permitted.

However, permission to reprint/republish this material for advertising or promotional purposes 


\title{
Millimeter Wave E-plane Transition from Waveguide to Microstrip Line with Large Substrate Size Related to MMIC Integration
}

\author{
A. Uz Zaman, Member, IEEE, V. Vassilev, and H. Zirath, Fellow, IEEE
}

\begin{abstract}
This paper demonstrates a packaging concept where a waveguide to microstrip transition can be assimilated on a mmwave MMIC of an arbitrary size and thus avoid the use of bond wires at the high frequency ports of the MMIC circuit. The advantage of the proposed concept is that- it does not require an extra substrate with sub-critical dimensions for the waveguide to microstrip transition. The transition can be integrated onto the MMIC and thus the MMIC can be coupled directly to the waveguide. A Perfect Magnetic Conductor (PMC) surface is used to suppress the unwanted waveguide mode coupling to the oversized circuit cavity. The measured back to back transition works over the frequency band $66-98 \mathrm{GHz}$ (relative $\mathrm{BW}$ of $38 \%$ ) with minimum return loss of $12.7 \mathrm{~dB}$. The total insertion loss of the manufactured prototype is found to be $3.26 \mathrm{~dB}$ which also includes a $14.8 \mathrm{~mm}$ long microstrip line on an Alumina substrate. The losses in a single transition vary between 0.25-0.42 dB.
\end{abstract}

Index Terms - E-plane probe, Perfect magnetic conductor (PMC) packaging, Perfect electric conductor (PEC), Overmoded cavity, etc.

\section{INTRODUCTION}

$\mathrm{E}$ MERGING millimeter-wave applications such as highresolution imaging, high-speed wireless data links, short-range radar and space applications will require low-loss, low cost and high-performance RF modules. Many millimeter-wave systems and modules are based on monolithic microwave integrated circuits (MMIC) built using microstrip transmission lines. Although MMICs are the essential components, devices such as antennas, high-qualityfactor (HQ) filters based on waveguide technology are also required. Therefore, a microstrip-to-waveguide transition is also essential element to interconnect these circuits. Several kinds of microstrip-to-waveguide transitions have been proposed in the millimeter-wave band. Among these transitions, a microstrip probe entering waveguide through a window or channel at its broad sidewall, commonly known as

Manuscript received September, 2015. This work is supported by European Research Council (ERC) via an advanced investigator grant ERC-2012ADG_20120216 and Swedish Governmental Agency for Innovation Systems (VINNOVA) within the VINN Excellence Center Chase at Chalmers.

A. Uz Zaman and P.-S. Kildal are with department of Signals and Systems, Chalmers University, SE-412 96 Göteborg, Sweden. Vessen Vassilev and Herbert Zirath are with the department of Microtechnology and Nanoscience at Chalmers University of Technology, (e-mail: zaman@ chalmers.se; per-simon.kildal@chalmers.se; vessen.vassilev@chalmers.se).
E-plane probe transition [1-3], has been used extensively due to wideband performance and modular simplicity.

However, this type of transition suffers from some drawbacks. The dimensions of the channel that holds the microstrip substrate and the E-plane probe must have very small width and height so that all the waveguide modes are under cut-off and only the microstrip mode can propagate within the band of operation. In case of high permittivity $\left(\varepsilon_{\mathrm{r}}>10\right)$ MMIC substrates such as typical GaAs or InP substrate, this waveguide channel width should be smaller than $0.43 \mathrm{~mm}$ at W-band as $\lambda \mathrm{g} / 2$ is $0.43 \mathrm{~mm}$ at $100 \mathrm{GHz}$. However, due to practical reasons, it is almost impossible to keep the size of a highly integrated multi-functional MMIC in that $0.43 \mathrm{~mm}$ range. Apart from that, it is also not realistic to laser cut or shape particular side of the delicate MMIC substrate $(50-100 \mu \mathrm{m}$ thick), as the narrower substrate part hanging inside the waveguide channel will not be able to handle mechanical stress and strain. Thus, a direct integration of a multi-functional MMIC to a rectangular waveguide is still a big concern at the mmWave frequency range. In this regard, the most common practice is to use an extra piece of substrate with sub-critical dimension for the microstrip probe and to place it in the narrow waveguide channel as an extended part of main MMIC [4]. The main MMIC is usually placed in a separate cavity and the RF output of the MMIC is electrically connected to the microstrip probe by bond wires. This is shown in Fig.1

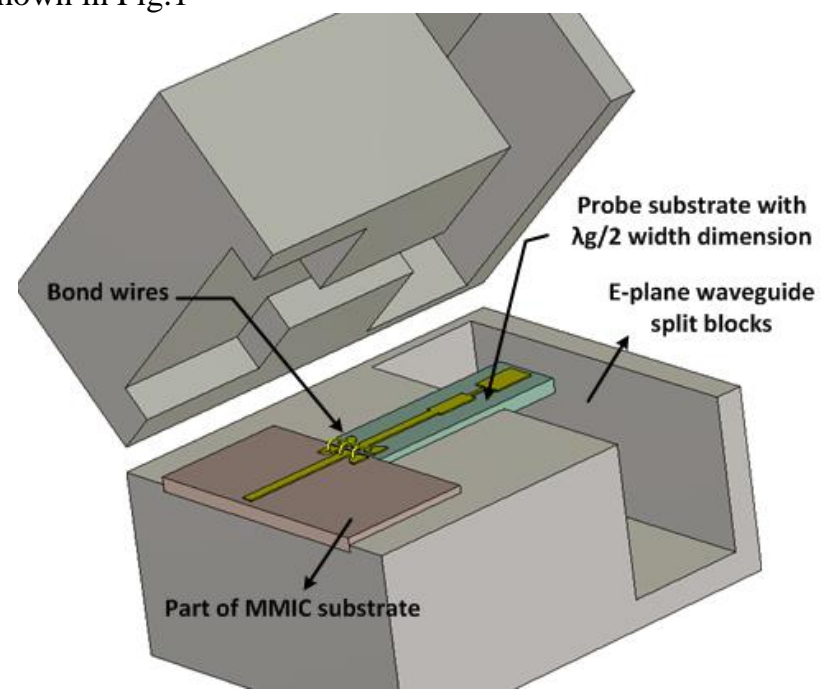

Fig. 1 Waveguide split blocks and typical MMIC integration scheme with bond wires. 
Unfortunately, parasitic effects such as high series inductance and unwanted radiation from bond wires make RF bonding at millimeter or sub-millimeter wave frequency range very unattractive. Also, bonding to a small size high frequency RF pads is associated to repeatability issue; hence there is a reduction in yield and increased manufacturing cost. As a consequence, RF engineers would like to integrate high frequency MMICs to the waveguide sections without any RF bond wires; in a way it would be ideal to put the whole MMIC with the probe inside the waveguide split-blocks. In this work, we propose a novel solution to the above mentioned problem and we use the E-plane probe with an oversized substrate inside the waveguide split-block without allowing any higher order mode coupling to the substrate. At this moment, we have validated the concept with passive back to back E-plane probe transition with oversized substrate. The measured results agree well with the simulation and we have demonstrated unwanted higher order mode suppression over a significant bandwidth.

\section{Proposed E-Plane Probe transition With PMC LID}

The proposed E-plane transition is very similar to the one presented in [1] except that the substrate size is intentionally kept much bigger $\left(8-10 \lambda_{\mathrm{g}}\right)$ in width. In this transition, a high impedance inductive line is first used in series with the probe to cancel out the capacitive reactance. After that, a quarterwave impedance transformer is used to match the real part of the probe impedance to $50 \Omega$ microstrip line. The substrate is soldered on the bottom split-block and can be of any thickness between 50-125 $\mu \mathrm{m}$.

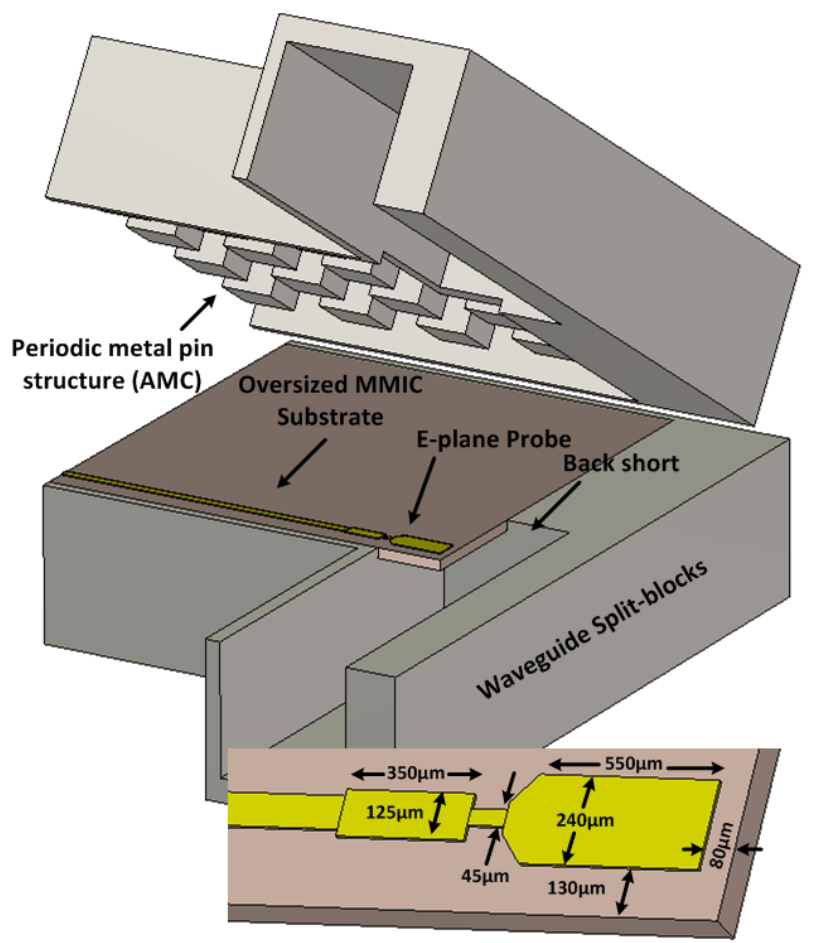

Fig.2 Schematic of the proposed transition with metal pin lid.

On the top split-block, we have used a periodic metal pin structure which acts as a perfect magnetic conductor (PMC) and changes the boundary conditions inside the bigger cavity with the substrate and suppresses all unwanted higher order cavity modes without disturbing the desired microstrip mode. This theory of suppressing higher order parallel-plate modes inside an oversized metal cavities lies on the fact that, in a PEC-PMC parallel-plate configuration, all EM waves will be under cutoff if the spacing between the PEC and PMC layer is kept smaller than $\lambda / 4[5,6]$. This mode suppression technique is also known as PMC packaging and can be very useful in microstrip circuits packaging and is mentioned in $[7,8]$. The schematic of the transition is shown in Fig.2.

In case of PMC packaging design, the metal pin dimensions are chosen in a way that the periodic pin structure exhibits a parallel-plate stopband within the band of interest. This is usually done by looking at the dispersion diagram of a single pin unit cell within a periodic boundary condition enforced around the unit cell. Fig. 3 shows the dimensions of the designed pin to be used in this work and also the computed dispersion diagram for the single pin unit cell.

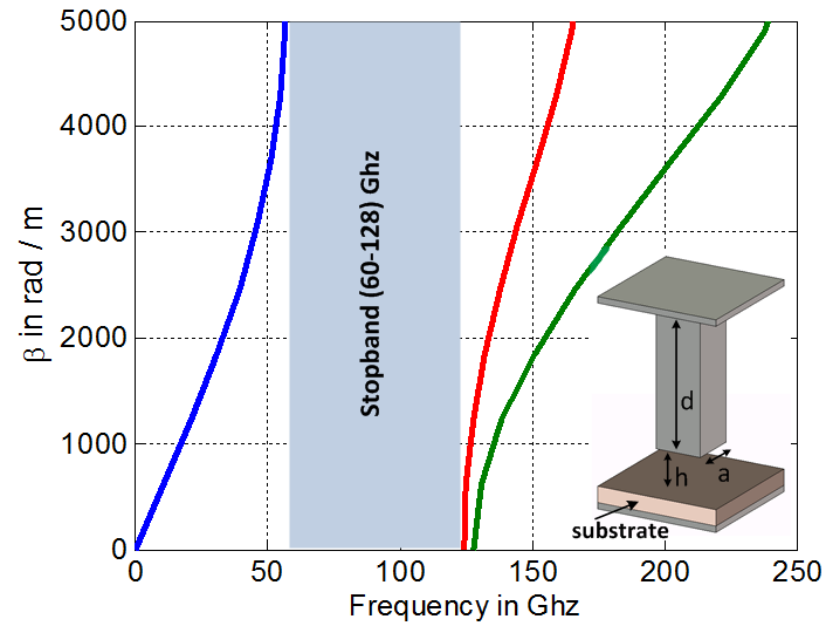

Fig.3 Dispersion diagram of the pin; $\mathrm{a}=0.45 \mathrm{~mm}, \mathrm{~d}=0.84$ $\mathrm{mm}$, pin period $=1 \mathrm{~mm}$ and $\mathrm{h}=0.135 \mathrm{~mm}$, substrate thickness $=125 \mu \mathrm{m}$.

\section{Simulated AND MeAsUREd ResUlts FOR THE TRANSITION}

The single transition has been designed at W-band and simulated first with both the smooth metal top and top block with periodic metal pins. The height between the smooth metal lid and the substrate has been kept $0.25 \mathrm{~mm}$ not to affect the microstrip line impedance. In case of the periodic metal pin, we have kept $0.135 \mathrm{~mm}$ gap between the pins and the substrate. The simulated $S_{11}$ for the proposed transition is presented in fig.4. We can clearly see the improvement in the $\mathrm{S}_{11}$ with the metal pin top block compared to the smooth metal top block when an oversized substrate has been used with the E-plane probe.

In order to validate the design, a back-to-back E-plane probe transition with periodic metal pin lid has been fabricated. We have used Alumina substrate $\left(\varepsilon_{\mathrm{r}}=9.9\right)$ from American Technical Ceramics (ATC) with $125 \mu \mathrm{m}$ substrate thickness. The total substrate size is $11 \times 4.5 \mathrm{~mm}^{2}$ and on this substrate a microstrip line with two $90^{\circ}$ bends has been printed along with the two E-plane probes. The total length of the microstrip line is $14.8 \mathrm{~mm}$. The measured result and the manufactured prototype are presented in Fig. 5 and Fig. 6 respectively. 


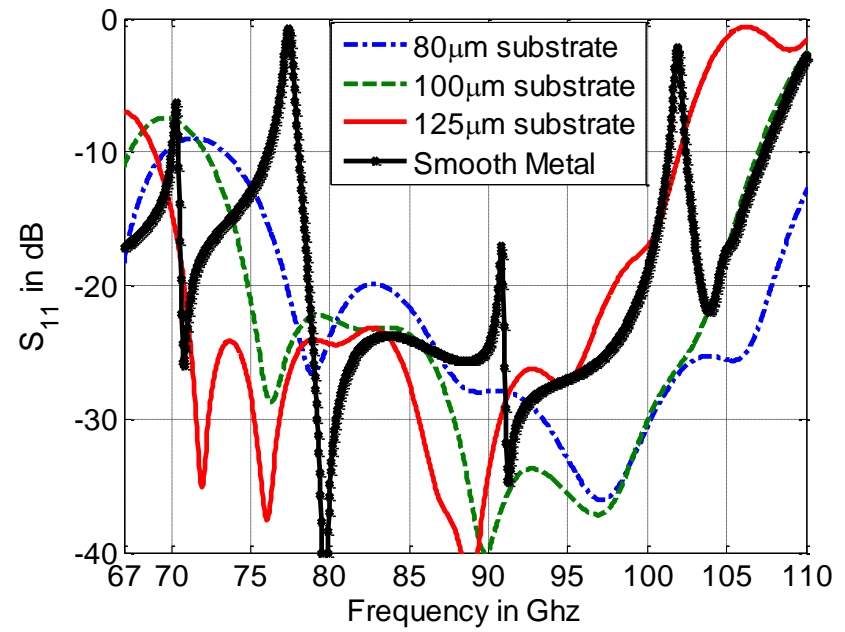

Fig. 4 Simulated $S_{11}$ results for single transition.
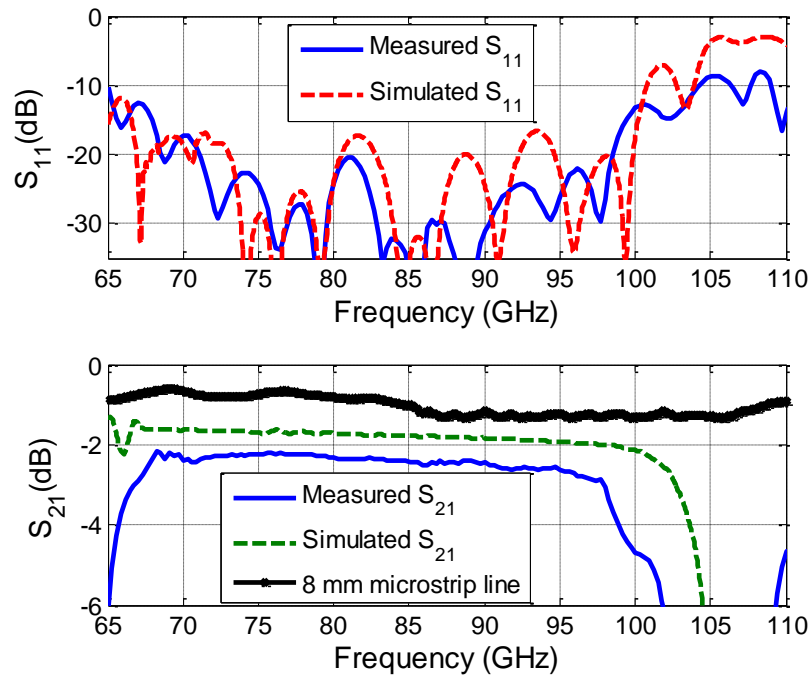

Fig. 5 Measured S-parameter results for the back-to-back E-plane probe transition with oversized substrate.

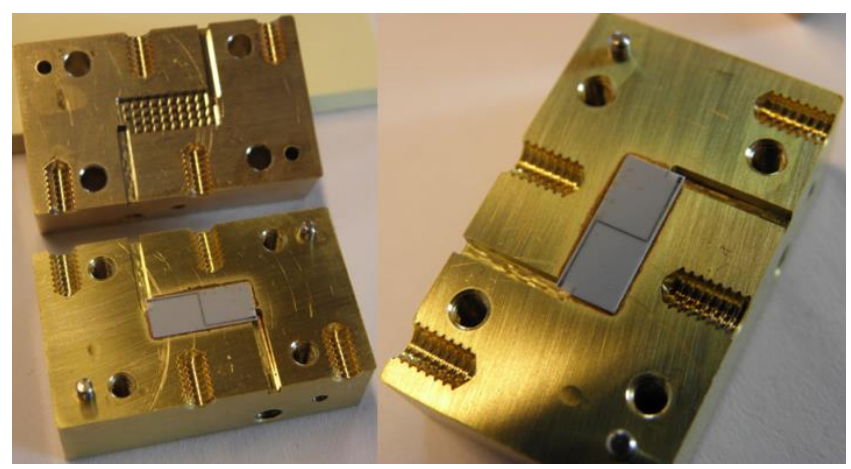

Fig. 6 Manufactured back-to-back transition with metal pin lid.

Measured back-to-back prototype is found to work within 66-98 GHz (BW: 38\%) with reasonable $\mathrm{S}_{11}$ and $\mathrm{S}_{21}$ parameters. The measured $S_{11}$ is also consistent with its simulated values at frequencies $66-98 \mathrm{GHz}$. The discrepancy between the simulated and measured $S_{11}$ is attributed to mechanical assembly errors which is quite critical at W-band. The measured $\left|S_{21}\right|$ of the back-to-back prototype is less than $3.26 \mathrm{~dB}$ over the entire $66-98 \mathrm{GHz}$ frequency range and follows well the trend of the simulated $S_{21}$. We also want to mention here that the $S_{21}$ here consists of both the transition losses and the losses in the $14.8 \mathrm{~mm}$ long microstrip line with two $90^{\circ}$ bends. The discrepancy between the simulated and measured $S_{21}$ is mainly due to copper surface roughness in the microstrip line and roughness of brass used in waveguide blocks. We have measured also a simple $8 \mathrm{~mm}$ long microstrip line on the similar Alumina substrate by GSG probing and we have estimated the losses in a $14.8 \mathrm{~mm}$ long microstrip line. After subtracting those losses of the microstrip line, total losses in a single E-plane probe transitions are found to be varying between $0.25-0.42 \mathrm{~dB}$. It is also important to remind that, no unwanted mode coupling occurs within the band of interest and the $S_{21}$ remains quite flat over a relatively large bandwidth.

\section{CONCLUSION}

In this paper, we have demonstrated a millimeter wave Eplane probe transition from microstrip to rectangular waveguide where the probe is located in an oversized substrate and the entire substrate can be inserted in the waveguide channel. The problem of unwanted mode coupling between the waveguide and the substrate has been stopped by using $\lambda / 4$ high metallic pin structure which works as PMC over specific frequency range. The measured results show low insertion loss, good return loss, broadband nature, and stable performance for the back-to-back prototype of the proposed transition. This novel transition scheme is very suitable for a multifunctional MMIC integration where the substrate size is much larger than the critical waveguide channel width and the integration can be performed without the need of RF bonding which is a big advantage compared to today's state of the art technique.

\section{REFERENCES}

L. Yoke-Choy and S. Weinreb, "Full band waveguide-tomicrostrip probe transitions," in Microwave Symposium Digest, 1999 IEEE MTT-S International, 1999, pp. 1435-1438 vol.4.

E. S. Li, T. Gui-Xiang, and N. Dow Chih, "Full W -band Waveguide-to-microstrip Transition With New E-plane Probe," Microwave and Wireless Components Letters, IEEE, vol. 23, pp. 46, 2013.

[3] C. Risacher, V. Vassilev, A. Pavolotsky, and V. Belitsky, "Waveguide-to-microstrip transition with integrated bias-T," Microwave and Wireless Components Letters, IEEE, vol. 13, pp. 262-264, 2003.

[4] V. Vassilev, N. Wadefalk, R. Kozhuharov, M. Abbasi, S. E. Gunnarsson, H. Zirath, et al., "MMIC-Based Components for MMWave Instrumentation," Microwave and Wireless Components Letters, IEEE, vol. 20, pp. 578-580, 2010.

[5] P. S. Kildal, A. U. Zaman, E. Rajo-Iglesias, E. Alfonso, and A. Valero-Nogueira, "Design and experimental verification of ridge gap waveguide in bed of nails for parallel-plate mode suppression," Microwaves, Antennas \& Propagation, IET, vol. 5, pp. 262-270, 2011.

[6] P. S. Kildal, E. Alfonso, A. Valero-Nogueira, and E. Rajo-Iglesias, "Local Metamaterial-Based Waveguides in Gaps Between Parallel Metal Plates," Antennas and Wireless Propagation Letters, IEEE, vol. 8, pp. 84-87, 2009.

[7] E. Rajo-Iglesias, A. U. Zaman, and P. S. Kildal, "Parallel Plate Cavity Mode Suppression in Microstrip Circuit Packages Using a Lid of Nails," Microwave and Wireless Components Letters, IEEE, vol. 20, pp. 31-33, 2010.

[8] A. Kishk, A. Uz Zaman, and P.-S. Kildal, "Numerical Prepackaging with PMC lid - Efficient and Simple Design Procedure for Microstrip Circuits including the Packaging," ACES Applied Computational Society journal, vol. 27, no.5, pp. 389-398, May 2012. 